\title{
Stage II Jejunal Neuroendocrine Tumor AJCC v8
}

National Cancer Institute

\section{Source}

National Cancer Institute. Stage /I Jejunal Neuroendocrine Tumor A/CC v8. NCI

Thesaurus. Code C135121.

Stage II includes: (T2, N0, M0); (T3, NO, M0). T2: Tumor invading muscularis propria or measuring more than $1 \mathrm{~cm}$ in size. T3: Tumor invading through the muscularis propria into subserosal tissue without penetration of overlying serosa. N0: No regional lymph node metastasis. M0: No distant metastasis. (AJCC 8th ed.) 